\title{
Spatio-temporal comparison of agent trajectory with evacuation routes in real and virtual environments
}

\author{
Dajana Snopkováa, ${ }^{\text {, }}$, Ondřej Uhlík ${ }^{\mathrm{b}}$, Ondřej Kvarda ${ }^{\mathrm{a}}$, Zdeněk Stachoň ${ }^{\mathrm{a}}$, Petr Kubíček ${ }^{\mathrm{a}}$ \\ ${ }^{a}$ Department of Geography, Faculty of Science, Masaryk University, Brno, snopkova.dajana@mail.muni.cz, 451448@mail.muni.cz, \\ zstachon@geogr.muni.cz, kubicek@geogr.muni.cz \\ ${ }^{b}$ Institute of Computer Aided Engineering and Computer Science, Faculty of Civil Engineering, Brno University of Technology, \\ ondrej.uhlik@vut.cz. \\ * Corresponding author
}

Keywords: evacuation, locomotion, wayfinding, agent simulation, virtual reality, user study

\begin{abstract}
:
Agent evacuation models are currently often used to simulate evacuation from buildings. In these models, algorithms for calculating the shortest distance are mainly used for motion simulation, or smoothing algorithms are applied for a more natural course of trajectories (Johnson, 2006; Reynolds, 1999). However, the trajectories of human movement do not fully correspond to these calculations, and the differences are especially noticeable on a larger (cartographic) scale at the level of buildings. In addition, the trajectories and the dynamics of movement are influenced by decision-making processes. During an evacuation, a significant effect of (not) noticing evacuation signs (Proulx, 2001, Johnson, 2005, Xie et al., 2011), or an application of the retracing navigation strategy (return by the same route) (Proulx, 2001, Kurkjian, et al. 2003, Johnson, 2005, Snopková, et al., 2021) is evident. The safe capacity of buildings is assessed in the design stage before they are actually built. Where it is impossible to perform evacuation drills, virtual reality seems to be a suitable substitution tool. Virtual environments dispose of a high level of realism, ensure high ecological experimental validity, and at the same time the possibility of non-invasive collection of behavioral data.
\end{abstract}

This contribution compares evacuation trajectories between the Pathfinder agent-based model and human evacuation in a virtual environment and an actual building. The comparison is a partial result of a larger user study focused on navigation behavior differences between real and virtual environments. In the user study, after entering the room, the participants were assigned a distraction task, during which a fire alarm sounded, and they had to find a way out of the building. In the real building, the position of 35 participants (15 men, 20 women) was recorded via a GoPro camera. In the virtual environment displayed using the HTC Vive headset and Unity graphic engine, data for 38 participants (22 men, 16 women) was logged in the background using Unity logger scripts and the Toggle Toolkit (Ugwitz et al., 2021). In the real environment, participants were instructed to evacuate with quick steps, not running. In the virtual environment, participants navigated through the environment using a keyboard and mouse at a constant speed of $3 \mathrm{~m} / \mathrm{s}$.

Additionally, we used the same building to run a single agent evacuation simulation in Pathfinder. Pathfinder is an agentbased numerical model with primary use in evacuation modeling. The model consists of a simulation core, graphical unit interface, and interface for analysis and 3D visualization. Simulation core calculates new positions of agents based on their profile (physical parameters of agents, door choosing behavior, distancing restrictions) and surrounding conditions (position of surrounding agents, time required for reaching room exit, etc.) every predefined time-step (Thornton et al., 2011). The user sets profile parameters for every agent or group of agents, which allows creating some known human behavioral patterns, e. g-choosing a shorter route to the final exit at the cost of longer time spent in congestions. Numerical results of the simulation are stored in standard text file formats enabling further processing. Two movement modes are present in the model (Thornton et al., 2011). In SFPE mode, agents make no attempt to avoid one another and can interpenetrate, but doors impose a flow limit, and walking speed is controlled by density (SFPE, 2019). STEERING mode (used in the presented model) tries to emulate human behavior and movement, which means that agents can reflect their surrounding geometry and other agents (Reynolds, 1999). Other relevant input parameters of the presented model in the context of this study are time-step: $0,025 \mathrm{~s}$, diameter: $46 \mathrm{~cm}$, walking speed on the horizontal plane: $1,19 \mathrm{~m} / \mathrm{s}$, speed of walking downstairs: $1,07 \mathrm{~m} / \mathrm{s}$, wall boundary distance: $0,15 \mathrm{~m}$. The trajectory route of agents was defined by the user allowing no decision-making. Input parameters were set based on data presented in (SFPE, 2019).

Due to the different recording methods and the resulting different temporal and spatial resolution, it was first necessary to harmonize the data collected for participants' passage through the virtual and real environments. Afterward, we used spatial relational databases and GIS processing tools to analyze spatiotemporal variations and movement dynamics between the two experimental conditions and the agent-based simulation. Since each pass of the agent through the 
building model in the case of the Pathfinder simulation shows the same results (unless the input parameters change), the resulting trajectory was compared with the average trajectories for both experimental conditions. Average trajectories were constructed as centrelines - approximations of central tendencies of all input trajectories except those parts of trajectories that had significantly deviated from the rest of the sample (e.g., when participants got lost). Further, we calculated average trajectory length, average trajectory obliquity as a ratio of actual trajectory length divided by the straight-line length between endpoints of the trajectory, and the average distance of a trajectory from the nearest selected corner of a corridor/staircase. The preliminary results are summarized in Table 1. Figure 1 illustrates the course of the compared trajectories from the beginning of the evacuation until the first staircase.

Table 1. Calculated metrics comparing evacuation trajectories across agent simulation and real and virtual experimental conditions.

\section{real condition virtual condition agent simulation}

\begin{tabular}{lccr}
\hline Average trajectory length [m] & 147.66 & 147.08 & 136.61 \\
Average evacuation time [s] & 118 & 87 & 136 \\
Average trajectory obliquity & 3.63 & 3.62 & 3.37 \\
Average distance from the nearest corner [m] & 0.86 & 0.80 & 0.38
\end{tabular}

\section{4"Floor \\ Lengths of trajectories [m]: 94.43 | 93.52| 91.25

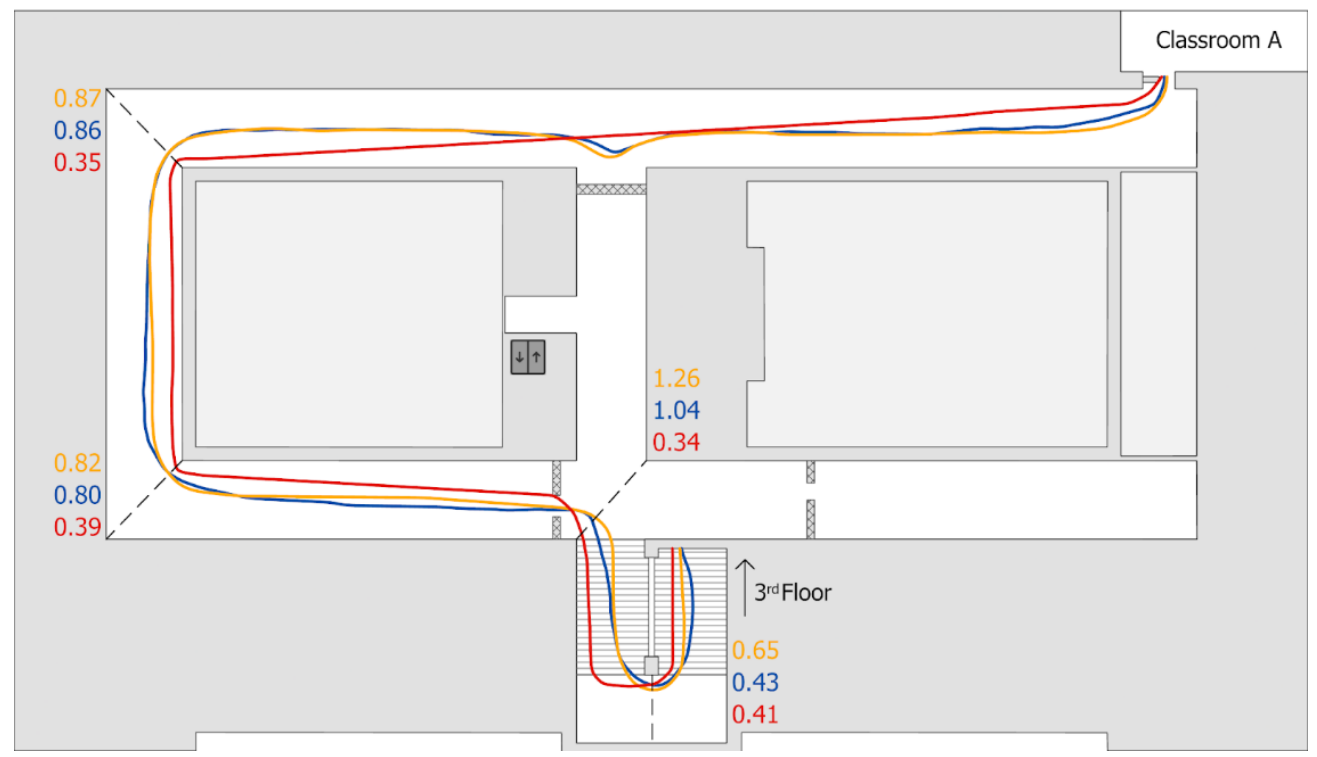

Figure 1. Visualization of compared evacuation trajectories across agent simulation and real and virtual experimental conditions.

Preliminary results show remarkable similarity between human evacuation routes in virtual and real environments. The average trajectory length and obliquity demonstrated the same values along with the average distance from the nearest corners. The only difference was identified in the evacuation time, which was significantly affected by the constant movement speed in the virtual environment and the absence of need for physical exertion. The agent's trajectory, on the other hand, differs from the remaining trajectories. The movement is more directional, and the agent moves closer to the walls, resulting from the input parameters setting.

The setting of the input parameters of the agent model was based on literature; however, this contribution represents empirically-based evidence that there is still room for improvement. At the same time, it confirms that in the context of trajectories, a virtual environment can be used to estimate human behavior in actual buildings. 